\title{
Planetesimal clusters in a Keplerian disk
}

\section{I. gravitational evolution}

\author{
P. Tanga ${ }^{1, \star}$, P. Michel $^{1}$, and D. C. Richardson ${ }^{2}$ \\ 1 Observatoire de la Côte d'Azur, BP 4229, 06304 Nice Cedex 04, France \\ 2 Dept. of Astronomy, Univ. of Maryland, College Park, MD 20742-2421, USA
}

Received 6 May 2002 / Accepted 30 August 2002

\begin{abstract}
It was recently demonstrated by numerical simulations that a turbulent flow in a rotating system is capable of efficiently concentrating passively advected particles - having a density larger than the fluid - inside anti-cyclonic vortices. This process has important consequences on the distribution of solid particles in protoplanetary disks, since dust surface densities $\sim 1-2$ orders of magnitude higher than the background are rapidly reached in vortex cores. However, until now, the role of self-gravitation of captured solids has been neglected. In this work we study the action of mutual gravitational interactions - after the gas has dissipated - over the dynamics of planetesimals inside clusters similar to those created in vortex cores. A comparison is made between the behavior of idealized clusters of planetesimals characterized by ad-hoc velocity profiles, and more complex initial conditions such as those obtained in previous hydrodynamical simulations. We show here that, within the explored interval of parameters, mutual scattering of particles can quickly disperse the cluster. Our results are demonstrated to be not dependent on the resolution employed. It can be concluded that if large planetesimals were formed inside vortex cores, they would be ejected by mutual perturbations.
\end{abstract}

Key words. planetary systems: formation, protoplanetary disks; gravitation

\section{Introduction}

One of the greatest difficulties in the study of Solar System formation is to understand in detail the growth of planetesimals, in particular in the phase during which gas drag is efficiently affecting particle dynamics. In recent years several theoretical and experimental studies have addressed this problem, starting from the stage of micrometer- or millimeter-sized dust (see e.g. Blum et al. 2000; Cuzzi et al. 2001).

It is well known that the presence of gas can strongly influence the dynamics of particles in the $1 \mathrm{~cm}-1 \mathrm{~m}$ range, resulting in radial migration and preventing gravitational instability (see e.g. Weidenschilling 1995; Cuzzi et al. 1993). So far, the classical approach used to study the accretion process has mainly been a statistical one: particles are considered to be uniformly distributed and their velocities are locally isotropic. The fact that the gaseous nebula is partially supported by pressure determines a rotation profile that is not Keplerian. Since pressure does not affect dust particles, these will tend to rotate at the Keplerian rate. The gas-solid velocity differential then causes small particles to drift toward the Sun and, due to their motion through the gas, small-scale turbulence is generated.

\footnotetext{
Send offprint requests to: $\mathrm{P}$. Tanga,

e-mail: tanga@obs-nice.fr

^ Financed by H. Poincaré fellowship.
}

When more realistic models of the effect of turbulent flows over gas and solids are taken into account, the distribution of transported particles does not remain uniform. Over several years, different studies have shown the properties of turbulent transport in both two and three dimensions. The particular case of particles much more dense than the surrounding fluid has been investigated in different contexts, suggesting particle segregation depending upon the local value of vorticity (see e.g. Squires \& Eaton et al. 1991; Crisanti et al. 1992; Dobrovolskis et al. 1993; Cuzzi et al. 1996). It has thus been verified that the presence of rotation inside coherent structures (vortices) is capable of deeply affecting a distribution of passively advected solids. This is true both in typical 3D scenarios, and in the case of large-scale vortices observed in rotating systems, where turbulence has the tendency to become essentially twodimensional (McWilliams 1999).

The possible survival of large-scale vortices in the Keplerian shear has been recently demonstrated by numerical simulations. In particular, it was shown that anti-cyclonic vortices could be present on the disk plane at large scales (with an upper limit typically of the order of the scale height of the disk, $H$ ), and survive over several revolutions, both in incompressible (Bracco et al. 1998) and compressible cases (Godon et al. 2000).

The role of vortices in Keplerian disks was revealed by numerical simulations performed using simple analytical models, 
either exact solutions of the incompressible Navier-Stokes equation (Tanga et al. 1996) or ad hoc representations (Barge et al. 1995). It was thus shown that centimeter- to meter-scale planetesimals can be efficiently captured by anti-cyclonic vortices and concentrated in their cores. Theoretical studies have further supported this scenario, clarifying the role of the friction parameter over the rate of mass capture (Chavanis 2000). The capture in vortices is due to the appearance of additional accelerations due to the global disk rotation, namely the Coriolis apparent force in a frame locally Co-rotating with the nebula.

Moreover, the study of convective motions perpendicular to the disk plane has shown that these kinds of circulation cells can also strongly affect the vertical dust distribution (Klahr et al. 1997), although by a completely different mechanism, based on the interplay between gravity and the vortical motion on a plane perpendicular to the disk.

It is thus clear that, in this context, simple assumptions such as uniformity and isotropy of the distribution of planetesimals are no longer valid, and that the usual statistics should be revisited in order to incorporate more complex effects. Of course, the real existence of these effects in the case of the Solar System has to be evaluated and supported in light of all the observational constraints. Nevertheless, particle capture in vortices is seen as an efficient mechanism for increasing particle growth rates, one that could really be active in some protoplanetary systems.

The construction of this scenario for planetesimal growth requires detailed studies of the gravitational interaction of captured particles. In fact, the motion of particles strongly coupled to the gas in vortices is fairly different from that of the highly chaotic, isotropic system of (locally) homogeneous density usually employed to study accretion rates. Relative trajectories of massless particles inside vortices have been found to be characterized by low values of the Lyapunov exponents, typical of regular systems (Babiano et al. 1994), due to the systematic, ordered motions that characterize vortex cores.

We can expect, however, that the presence of inter-particle gravity can break this order by mutual perturbations, introducing a diffusion that could eventually lead to the escape of particles from vortices. In fact, mutual gravitational scattering of particles has the tendency to destroy the memory of the initial velocity profile. Depending upon the relative contribution of gas drag and scattering, the competition between these two processes could lead to different results, such as the ejection of particles from the vortex or, on the contrary, a concentration in their core with an enhanced growth rate. Several complex processes have thus to be considered in order to derive a consistent scenario: beside mutual scattering, the contribution of gas-particle interactions, statistics of mutual collisions and relative velocities and impact outcomes have to be considered. In order to understand the role of each of these components, in this work we move a step forward by considering the effect of pure gravity on particles in clusters one or two orders of magnitude more dense than the disk background. Such cluster densities are consistent with the observed capture efficiencies. In a forthcoming paper, we will address the problem of a more complex scenario, taking into account gravity and gas at the same time.
In Sect. 2 we will describe an "ideal" cluster of planetesimals on a circular orbit around the Sun. We will show that, in the absence of mutual gravity between particles, it would never dissipate or change its structure. In Sect. 3 its dynamical evolution under the effect of mutual particle gravity will be investigated by means of $N$-body simulations. Finally, in Sect. 4 we will present a comparison with simulations in which initial conditions are provided by a more complex scenario.

\section{Model of a cluster of planetesimals: The ideal case}

A stability analysis of density fluctuations was performed by Safronov (1969) and Goldreich \& Ward (1973) in their classical model of planet formation. Later, Weidenschilling (1995) showed that the velocity dispersion in the typical "Solar Nebula" environment is far too high to allow gravitational instability to take place. However, it must be noted that in all those cases planetesimal velocities are considered to be completely uncorrelated.

On the other hand, when clustering is determined by trapping in vortices, we can expect a strong velocity correlation in the motion of planetesimals in high-vorticity domains. For this reason, we study here self-gravitating clusters whose initial velocities are strongly correlated. In the following, the term "cluster" (of planetesimals) will identify the peculiar density fluctuation generated by capture in vortices.

We assume, as a first step, that particles have already been captured in a vortex, and that, subsequently, they evolve under the effect of their pure mutual gravitational attraction, no longer affected by any gas drag. We assume also that the cluster of planetesimals resulting from this capture is in a circular orbit around the Sun.

The first challenge is to identify a simple velocity distribution satisfying two conditions: (a) the cluster has to remain constant in size, shape and total mass over several revolutions; (b) the distribution of planetesimals and their velocities must reflect the fundamental properties of the dynamics of particles captured in vortices.

Of course, if all planetesimals in the cluster have exactly the same value of their semi-major axis, their relative positions will be the same after each orbit (assuming here that their mutual interactions are negligible) and thus, for a very limited fraction of the orbit itself, the original clustering will be reproduced. However, in general, this solution remains unsatisfying since mutual gravitation between particles belonging to the cluster acts on some very short arc along the orbit. Furthermore, if orbital angles are not correlated, a "vortex-like" velocity profile cannot be identified, (as required by $[b]$ ). Also, such a cluster will reproduce its shape each revolution period, but it will not preserve it all along the orbit (condition $[a]$ ).

Therefore, if we want to reproduce the situation of particles that remain confined in a restricted region of space all along their orbit, appropriate choices of their eccentricities and orbital angles are required.

A simple solution can be easily found in a rotating reference frame centered on the cluster (in a circular orbit of radius $a_{\mathrm{c}}$ from the Sun) with average period $T_{\mathrm{c}}=2 \pi / \Omega$, where 


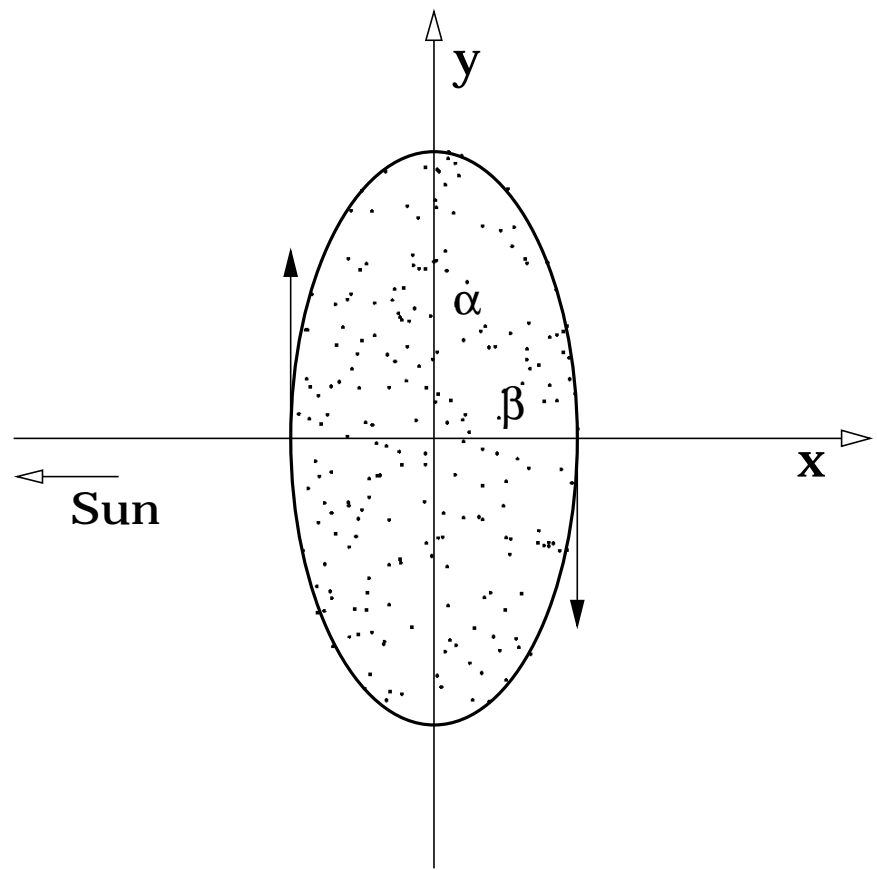

Fig. 1. The geometry of the solution for a particle trajectory in a local reference frame. The Sun is on the left. $\alpha$ and $\beta$ represent the semi-axes of the ellipse of the elliptical patch containing the cluster, indicated by dots.

$\Omega$ is the orbital frequency of the center of the cluster. The coordinate plane $(x, y)$ is coincident with the invariant plane of the system, with the $x$ axis oriented opposite to the Sun direction. If the cluster diameter is $d_{\mathrm{c}} \ll a_{\mathrm{c}}$, all particles belonging to it will have $x \ll a_{\mathrm{c}}$. In such conditions their equations of motion can be linearized.

Neglecting here the vertical component, the trajectory of an unperturbed particle on a Keplerian orbit obeys the equation (see e.g. Wisdom \& Tremaine 1988):

$\left\{\begin{array}{l}\dot{u}-2 \Omega \omega=0 \\ \dot{\omega}+\frac{1}{2} \Omega u=0 \\ \omega=v+v_{\mathrm{k}}\end{array}\right.$

in which $u$ and $v$ are the velocities along $x$ and $y$, respectively, and $v_{\mathrm{k}}$ is the linearized Keplerian shear $v_{\mathrm{k}}=\frac{3}{2} \Omega x$.

A particle that does not leave the cluster during its orbital motion will describe a closed trajectory in the chosen frame. For example, if at a given time an unperturbed particle with semi-major axis $a$ has $y=0, x=-\beta$ (with $\beta>0$ ) and $\dot{x}=0$ (see Fig. 1), then it will be at perihelion. With passing time, it will move toward larger values both in $x$ and $y$, until $x=0$. The motion along $y$ will then be inverted, and the particle will arrive at aphelion $(x=\beta)$ after a time $T_{\mathrm{c}} / 2$.

System (1) can be integrated analytically to find velocities and positions, as a function of time $t$. The first step provides:

$\left\{\begin{array}{l}u=2 \omega_{0} \sin (\Omega t) \\ \omega=\omega_{0} \cos (\Omega t)\end{array}\right.$ where $\omega_{0}$ is an integration constant. The general solution for the trajectory is:

$\left\{\begin{array}{l}x=x_{0}+\frac{2 \omega_{0}}{\Omega}[1-\cos (\Omega t)] \\ y=y_{0}-3 t\left(\frac{x_{0} \Omega}{2}+\omega_{0}\right)+\frac{4 \omega_{0}}{\Omega} \sin (\Omega t)\end{array}\right.$

in which we introduce the initial position $\left(x_{0}, y_{0}\right)$. From (2) and (3), for a given initial position there exists a single velocity assignment that produces a closed trajectory in the rotating frame, similar to that plotted in Fig. 1. This particular solution has $\omega_{0}=-x_{0} \Omega / 2$. It can be easily shown that the flattening ratio of the trajectory is $\alpha / \beta=2$. This solution corresponds to the so-called Guiding Center Approximation (see e.g. Murray et al. 1999).

Thus, if a cluster of particles with $\alpha / \beta=2$ is created, assigning to particles the velocities defined in Eq. (2), and mutual gravitational interactions are neglected, all particles will "orbit" around the cluster center with a systematic motion. The velocity profile mimics the rigid rotation usually observed inside vortex cores in $2 \mathrm{D}$ turbulence. If an initial dispersion along $z$ is imposed, at the same time the particles will oscillate vertically with frequency $\Omega$.

Since the projection of the cluster shape over the nebula plane will remain constant in time, we can say that this idealized solution corresponds to a stationary cluster for massless particles.

As a verification we performed an $N$-body simulation using massless particles, in the heliocentric frame. The size of the cluster (semi-major axis) was $R_{\mathrm{c}}=0.1 \mathrm{AU}$. Any departure from the given cluster shape could then be attributed to the linearization approximation, but none was observed over several tens of orbits. The cluster kept its shape and size throughout the simulation. Further, a rotation rate equal to $\Omega$ corresponds, if interpreted as the rotation of a vortex, to the low Rossby number (close to unity) necessary for efficient trapping (Tanga et al. 1996). We have thus used these idealized clusters as initial conditions of $\mathrm{N}$-body simulations in which mutual gravitation was taken into account.

\section{Gravitational evolution of "ideal" clusters}

In this section we consider "ideal" clusters whose initial surface density is uniform. The most important parameter studied here is the amplitude of the density fluctuation $\eta$, given by the ratio between the surface density of the cluster $\left(\Sigma_{c}\right)$ and the average surface density of a uniform disk at the same distance from the Sun $\left(\Sigma_{\mathrm{d}}\right): \eta=\Sigma_{\mathrm{c}} / \Sigma_{\mathrm{d}}$. The rate of dispersion of such structures and its dependence upon both the resolution (number of particles) and the initial local density amplification has been studied.

In order to perform simulations at the highest possible resolution, we used a parallel $N$-body hierarchical tree code (Richardson et al. 2000), previously ported to various platforms and fully tested ${ }^{1}$. This code is capable of detecting mutual collisions of particles considered as rigid spheres. In the

\footnotetext{
${ }^{1}$ In particular, simulations were performed on the 4-processor alpha machine SIVAM at Nice Observatory and the Cray T3E of IDRIS, France.
} 
Table 1. Simulations of cluster evolution and resulting dispersion times. $N_{\mathrm{cl}}$ and $N$ are the number of particles in the cluster and in the background disk. $N$ in parentheses correspond to simulations in which the background disk was neglected. $T_{70 \%}$ represents the time required to disperse $70 \%$ of the cluster particles, and expressed in $\operatorname{yr}(2 \pi)^{-1}$.

\begin{tabular}{cccccrrr}
\hline \hline$\#$ & $\eta$ & $a_{\mathrm{c}}$ & $R_{\mathrm{c}}$ & $N$ & $N_{\mathrm{cl}}$ & $\left\langle z_{\mathrm{H}}^{2}\right\rangle^{1 / 2}$ & $T_{70 \%}$ \\
\hline 1 & 10 & 5 & 0.1 & $10^{4}$ & 22 & 3 & 329.8 \\
2 & 10 & 5 & 0.1 & $\left(10^{4}\right)$ & 22 & 3 & 339.9 \\
3 & 10 & 5 & 0.1 & $\left(10^{5}\right)$ & 223 & 3 & 270.2 \\
4 & 10 & 5 & 0.1 & $10^{6}$ & 2232 & 3 & 278.3 \\
5 & 10 & 5 & 0.1 & $\left(10^{6}\right)$ & 2232 & 3 & 280.2 \\
6 & 10 & 5 & 0.1 & $\left(10^{7}\right)$ & 22333 & 3 & 280.2 \\
7 & 20 & 5 & 0.1 & $\left(10^{5}\right)$ & 446 & 3 & 180.3 \\
8 & 50 & 5 & 0.1 & $\left(10^{5}\right)$ & 1130 & 3 & 109.9 \\
9 & 50 & 5 & 0.1 & $10^{5}$ & 1130 & 3 & 109.3 \\
10 & 100 & 5 & 0.1 & $10^{3}$ & 23 & 3 & 74.8 \\
11 & 100 & 5 & 0.1 & $\left(10^{3}\right)$ & 23 & 3 & 69.7 \\
12 & 100 & 5 & 0.1 & $\left(10^{4}\right)$ & 236 & 3 & 69.7 \\
13 & 100 & 5 & 0.1 & $10^{5}$ & 2354 & 3 & 65.3 \\
14 & 100 & 5 & 0.1 & $\left(10^{5}\right)$ & 2354 & 3 & 69.7 \\
15 & 100 & 5 & 0.1 & $\left(10^{6}\right)$ & 23560 & 3 & 66.0 \\
16 & 10 & 5 & 0.1 & $\left(10^{5}\right)$ & 2354 & 3 & 219.9 \\
17 & 10 & 5 & 0.1 & $\left(10^{5}\right)$ & 2354 & 15 & 430.4 \\
18 & 10 & 5 & 0.1 & $\left(10^{5}\right)$ & 2354 & 30 & 500.1 \\
19 & 10 & 5 & 0.1 & $\left(10^{5}\right)$ & 2354 & 60 & 500.1 \\
20 & 10 & 5 & 0.05 & $\left(10^{7}\right)$ & 5584 & 3 & 210.0 \\
21 & 50 & 5 & 0.05 & $\left(10^{5}\right)$ & 283 & 3 & 65.2 \\
22 & 100 & 5 & 0.05 & $\left(10^{6}\right)$ & 5890 & 3 & 44.9 \\
23 & 100 & 5 & 0.025 & $\left(10^{6}\right)$ & 1473 & 3 & 32.0 \\
\hline & & & & & & &
\end{tabular}

simulations presented here each collision between two particles results in merging, i.e. in a single sphere having a mass equal to the sum of the involved masses and the same density. This is a physically reasonable choice since relative velocities of colliding particles are typically smaller than the mutual escape velocity. Nevertheless, since the rate of physical collisions during the time scales of interest remains very low, the particle growth rate is not studied at this stage.

The parameters of the different simulations are summarized in Table 1. Our results show that the cluster dissipate in all simulations. This could have been suspected by analyzing the dispersion relation of density fluctuations of size $\lambda$ in a Keplerian disk (written here following the notation of Ward 1976), in which the relative importance of angular momentum, self-gravity and velocity dispersion $c$ are considered:

$F(\lambda)=4 \pi^{2} c^{2}-4 \pi^{2} G \eta \Sigma_{\mathrm{d}} \lambda+\lambda^{2} \Omega^{2}$.

The numerical estimate of Eq. (4) shows that, for $\eta \sim 1$, the gravitational instability can be reached for perturbations having wavelengths several orders of magnitude smaller than the typical size $\left(R_{\mathrm{c}} \sim 0.1 \mathrm{AU}\right)$ of our clusters (Weidenschilling 1995).

In order to allow the gravitational term to prevail, thus reaching gravitational instability, a density of the order of $\eta \sim 10^{3}$ is necessary, provided a negligible velocity dispersion $c$, and $R_{\mathrm{c}} \sim 0.1$. Nevertheless, it must be noted that Eq. (4) is derived for small density fluctuations $(\eta \sim 1)$; furthermore, it is valid for isotropic velocity dispersions only, that is for a situation very far from that considered here. Despite these limitations, we can consider it to be still valid for the simple orderof-magnitude estimate given. If now we consider relative velocities initially dominated by the profile in Eq. (2), they will be small but not negligible. Furthermore, the most dense cluster we consider will have $\eta \sim 100$ thus, as a consequence of the estimate above, its self-gravity will not be sufficient to reach gravitational instability.

Since the cluster collapse is not possible, the mutual gravitational perturbations between particles break the strict order of the initial velocity distribution through an increase in $c$ and a consequent diffusion in orbital elements, resulting in the cluster dissipation.

We have thus studied the timescales of dispersion as a function of resolution, initial density amplification $\eta$, and vertical dispersion. We also give some hints about the role of cluster size $\left(R_{\mathrm{c}}\right)$. All simulations were run for a total time corresponding to several tens of revolutions of the cluster, thus allowing the study of the short-term dispersion of clusters of trapped particles. In general, the cluster is eventually dispersed in a ring of size $\sim R_{\mathrm{c}}$, slowly spreading all along the orbit and becoming increasingly uniform in heliocentric longitude. The study of the long term evolution of this purely radial feature, no longer connected to the original vortex capture, is outside the scope of this paper.

We distinguish between two classes of simulations: (1) a disk with a cluster at $a_{\mathrm{c}}=5 \mathrm{AU}$ from the Sun; (2) an isolated cluster at the same distance. The comparison between class (1) and (2) allows us to study the effect of the background disk on cluster dispersion. Class (2) simulations will be characterized by a fairly small number of particles $N_{\mathrm{cl}}$, typically up to $N_{\mathrm{cl}} \sim 2 \times 10^{4}$. Simulations of isolated clusters having $N_{\mathrm{cl}} \sim 10^{5}$ could be performed by the $N$-body code without any particular difficulty, but at the price of a much longer computation time spent in data reduction, when building the statistics that will be presented below. Furthermore, their significance for the conclusions reached below would be very limited. On the other hand, it was possible to reach the practical limits of the $N$-body simulations for class (1), with $N=10^{6}$.

For the largest part of the simulations we located the cluster at $a_{\mathrm{c}}=5 \mathrm{AU}$ from the Sun, and we imposed a size $\beta \equiv R_{\mathrm{c}}=0.1 \mathrm{AU}$, with uniform surface density; this is consistent with the vortex dimensions used in Tanga et al. (1996). The amplification factor $\eta$ was chosen to correspond to a moderate concentration in the vortex, and was computed relative to a disk of average density $\Sigma_{\mathrm{d}}=1.2 \mathrm{~g} \mathrm{~cm}^{-2}$, close to to the density of solids in the standard "Minimum Mass" Solar Nebula at 5 AU. Particle bulk density was assumed to be $2.7 \mathrm{~g} \mathrm{~cm}^{-3}$. For models with $N=10^{6}$ these parameters correspond to a planetesimal radius of $\sim 116 \mathrm{~km}$. Of course, this planetesimal size is needed to reproduce the desired surface density, given the maximum practical limit to $N$, and must not be considered strictly representative of the size of particles efficiently captured in vortices. Nevertheless, as we will show in the following, the particle size (i.e. the "resolution" of our $N$-body simulations) does not affect appreciably our results.

In Table 1 the vertical dispersion is represented by the dispersion in the direction $z$, perpendicular to the disk plane, 
normalized to the reduced Hill radius: $\left\langle z_{\mathrm{H}}^{2}\right\rangle^{1 / 2}$. The reduced Hill radius for the mutual interaction of particles of mass $m_{\mathrm{p}}$ and average distance $a$ from the Sun is:

$h=\left(\frac{2 m_{\mathrm{p}}}{3 M_{\odot}}\right)^{1 / 3}$.

The Hill radius is then given by $R_{\mathrm{H}}=a h$.

In the range of parameters explored, due to the large cluster size, the average spacing between particles is always much larger than $R_{\mathrm{H}}$. Assuming that the cluster is a cylinder of approximate height $2 a h\left\langle z_{\mathrm{H}}^{2}\right\rangle^{1 / 2}$, its volume can be written as $V \simeq 2 \pi R_{\mathrm{c}}^{2} a h\left\langle z_{\mathrm{H}}^{2}\right\rangle^{1 / 2}$. The average inter-particle distance is $<d>\simeq\left(V / N_{\mathrm{cl}}\right)^{1 / 3}$, where $N_{\mathrm{cl}}$ is the number of particles inside the cluster. Even for the cluster for which the ratio $\langle d\rangle / R_{\mathrm{H}}$ is the lowest (case 6) we have:

$$
\begin{aligned}
& R_{\mathrm{H}}=4.42 \times 10^{-4} \mathrm{AU}, \\
& <d>=3.02 \times 10^{-3} \mathrm{AU} .
\end{aligned}
$$

The average time interval required for a single particle to encounter another one at a distance smaller than $R_{\mathrm{H}}$ (assuming that the cluster does not disperse) can be estimated from: $\tau_{\text {ce }} \simeq V\left(\pi R_{\mathrm{H}}^{2} v_{\text {rel }} N_{\mathrm{cl}}\right)^{-1}$, in which $v_{\text {rel }}$ is the relative velocity of the two interacting bodies. Under the hypothesis of a slow (quasi-stationary) evolution, we can use the rigid profile $(2,3)$ to estimate the relative velocity of two particles separated (on the plane) by the distance $2 R_{\mathrm{H}}$ :

$v_{\text {rel }} \simeq \Omega R_{\mathrm{H}}$.

This simple relation will be valid, at least, at the beginning of the evolution of the system. From the example above we get $\tau_{\text {ce }} \simeq 0.016 \mathrm{yr} \ll T_{\mathrm{c}}$, indicating that, despite the large distance $\langle d\rangle$, the system cannot be considered as strictly collisionless. We thus expect the cluster to evolve under the effect of the gravitational perturbations between particles. This result is obtained by considering the horizontal velocity only, thus discarding any contribution coming from the motion on a direction perpendicular to the disk plane. Anyway, if we also take into account an additional vertical component, the quantitative estimate could still change, but only in the direction of even smaller values of $\tau_{\mathrm{ce}}$.

\subsection{Results}

The typical evolution of a cluster is shown in Fig. 2. In the following we detail the effects of mutual perturbations. They induce a dispersion in the semi-major axis values, resulting in a progressive cluster dissipation, due to the Keplerian shear. The dissipation rate of the cluster can be estimated by the rate of mass loss, i.e. by the fraction of particles leaving the original cluster boundaries. The value of $T_{70 \%}$ (the time required for dissipating $70 \%$ of the original particle number $N_{\mathrm{cl}}$ ) gives a useful indication of the dispersion rate, and is listed in Table 1. Other parameters could be chosen as indicators, but it can be verified that the results exposed in the following would not change.
First, we have found that in the parameter space explored the presence of a background disk is completely negligible during the short-term evolution of the cluster. For this reason a large fraction of our simulations concerns a system including only the Sun and an isolated cluster. When included, the disk has been simulated by an annulus of planetesimals extending from 4.5 to $5.5 \mathrm{AU}$, with a vertical dispersion equal to that of the cluster, and a radial surface density profile $\Sigma(r) \sim r^{-1.5}$.

A first remarkable result is that the dispersion rate $T_{70 \%}$ is essentially independent of the chosen resolution (number of particles $N$ ), as long as all the other parameters (vertical dispersion, cluster mass) are kept constant. This has been checked for $\eta=10$ (simulations $1-6$ ) and $\eta=100$ (simulations 10-15). In both cases the number of particles in the cluster was varied over four orders of magnitude, without noticeable changes. The only small discrepancy (a difference of 20\%) for cases Nos. 1 and 2 is not significant since the number of particles in the cluster is too small $\left(N_{\mathrm{cl}} \sim 20\right)$ to provide meaningful statistics.

Even a more detailed analysis of the cluster dispersion does not reveal any significant difference. We plotted in Fig. 3 (upper panel) the number of particles remaining in the cluster (normalized to the initial value $N_{\mathrm{cl}}(0)$ ), as a function of time, for three different resolutions, for a cluster having $\eta=10$ at the beginning. The behavior of the three systems is substantially identical, and systematic differences are negligible if compared to the overall trend.

In order to test the dynamics more deeply, we computed the average quadratic velocity deviation relatively to the initial velocity profile (2). In other words, calling $u_{\mathrm{p}}, \omega_{\mathrm{p}}$ the radial and tangential components of the velocity of a particle at a given time, referred to the cluster center, we define the corresponding dispersions and the total dispersion, as:

$$
\begin{aligned}
& \left\langle u^{2}\right\rangle^{1 / 2}=\left(\frac{\sum\left(u_{\mathrm{p}}-u\right)^{2} m_{\mathrm{p}}}{\sum m_{\mathrm{p}}}\right)^{1 / 2} \\
& \left\langle\omega^{2}\right\rangle^{1 / 2}=\left(\frac{\sum\left(\omega_{\mathrm{p}}-\omega\right)^{2} m_{\mathrm{p}}}{\sum m_{\mathrm{p}}}\right)^{1 / 2} \\
& \left\langle u_{\mathrm{tot}}^{2}\right\rangle^{1 / 2}=\left(\frac{\sum\left[\left(u_{\mathrm{p}}^{2}+\omega_{\mathrm{p}}^{2}\right)^{1 / 2}-\left(u^{2}+\omega^{2}\right)^{1 / 2}\right]^{2} m_{\mathrm{p}}}{\sum m_{\mathrm{p}}}\right)^{1 / 2}
\end{aligned}
$$

where $u, \omega$ are the radial and tangential velocities referred to the cluster center that a particle obeying to the profile defined by Eq. (2) would have. The sums are performed over all particles inside the cluster. Correctly, the graph in Fig. 3 (middle panel) shows that at $t=0$, no dispersion is present relative to the theoretical profile. The subsequent evolution again does not show any dependence upon resolution.

Finally, the dispersion in semi-major axis $a$, inclination and eccentricity of particles inside the cluster was computed. While inclinations and eccentricities remain always close to their initial values, the evolution of $a$ presents some interesting features (Fig. 3, lower panel). At $t=0$ for all particles we have $a=a_{\mathrm{c}}$, as imposed by initial conditions, so $\left\langle a^{2}\right\rangle^{1 / 2}=0$. The increase of $\left\langle a^{2}\right\rangle^{1 / 2}$ at the beginning of the evolution is closely related to the most rapid growth of the velocity dispersions (middle panel). The population of particles whose semi-major axis is more distant from the original $a_{\mathrm{c}}$ will leave the cluster area more rapidly. The dynamical evolution thus gradually selects 

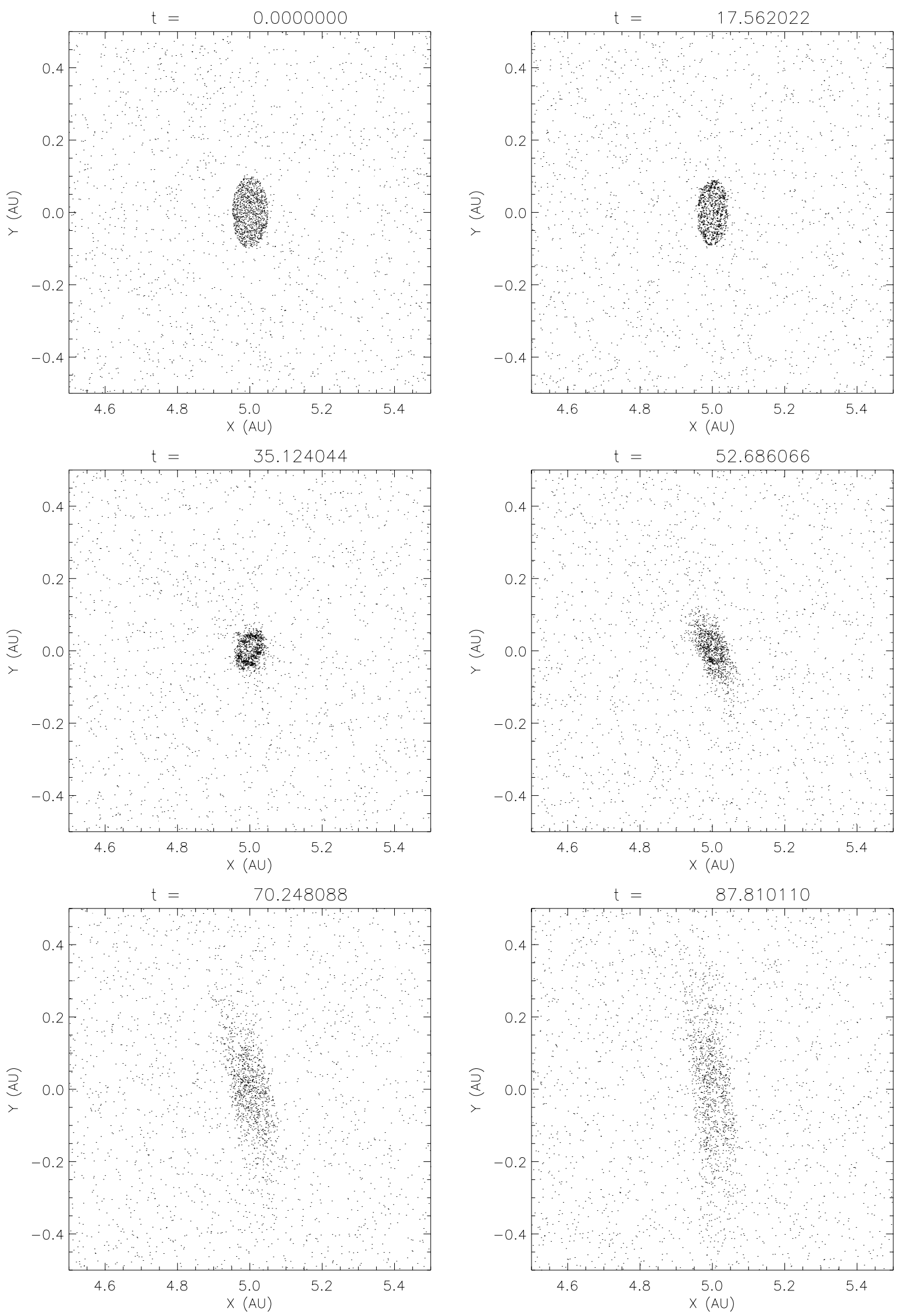

Fig. 2. A portion of the planetesimal disk of simulation No. 9 centered on the cluster, at the indicated times (in units of $\left.y r(2 \pi)^{-1}\right)$. The total number of particles in the disk is $10^{5}$. The first phase of the evolution is shown (running left to right, top to bottom). The origin of the $x$ axis is at the Sun position, while that of $y$ is fixed to a point co-rotating with the cluster center. 

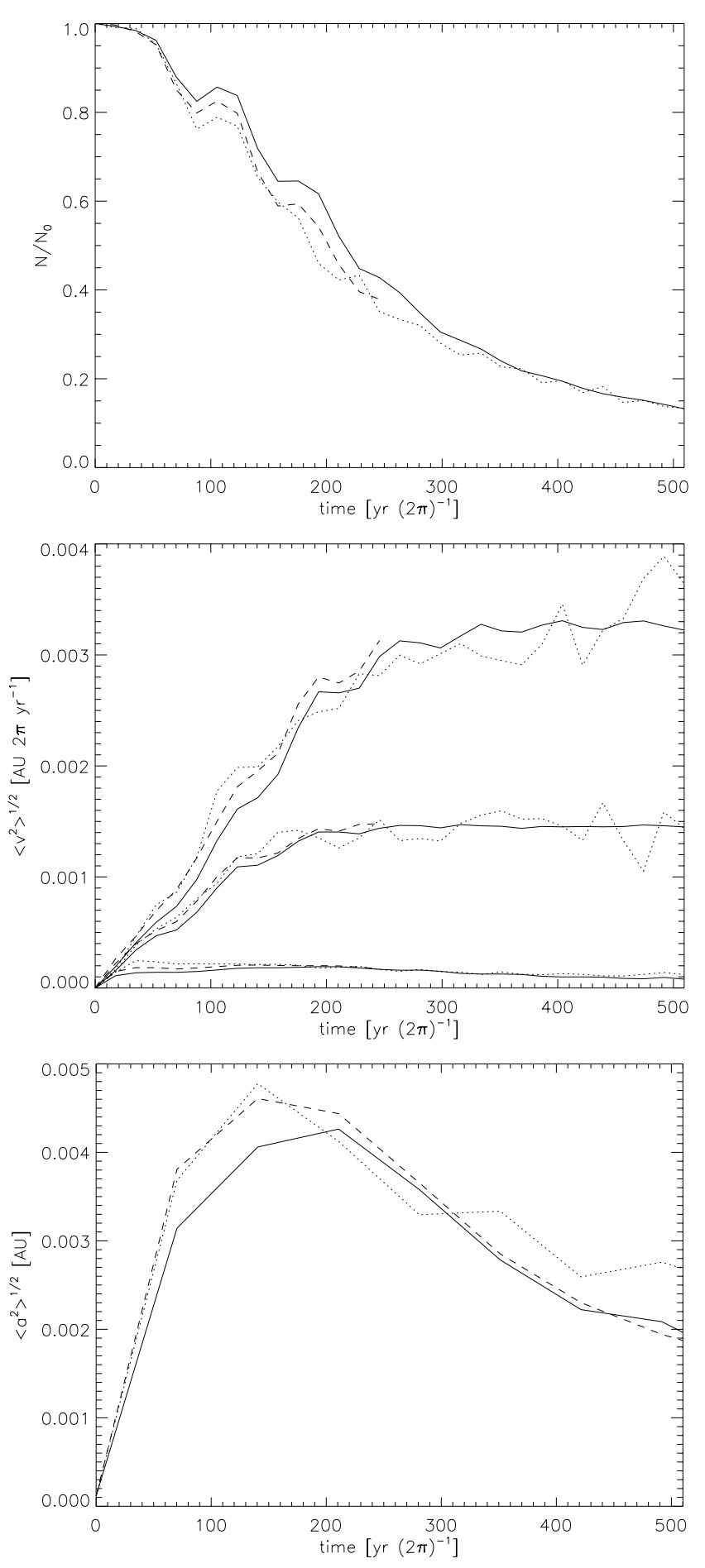

Fig. 3. Time evolution of some statistically relevant quantities in simulations Nos. 3 (dotted line), 5 (dashed), 6 (solid line). The upper panel shows the number of particles inside the original elliptic area, centered on a circle at distance $a_{\mathrm{c}}$ and having the corresponding Keplerian velocity around the Sun. The value is normalized to the initial value $N_{0}$. The middle panel shows the behavior of the average deviation from the initial velocity profile as defined by (8). The three curves correspond (upper to lower) to the deviations of the radial and total components (referred to the cluster center), and to that of the norm, respectively. Despite a variation in $N$ of over two orders of magnitude, the dynamics of the system is only marginally affected by the change in resolution. Finally, in the lower panel, the semi-major axis dispersion is plotted. At the beginning, for all particles $a=a_{\mathrm{c}}$. those particles that have a value of $a$ that remains progressively closer to the original $a_{\mathrm{c}}$. For this reason, after the initial "heating", the dispersion of semi-major axis decreases. It becomes nearly stationary toward $t=10^{3}$ (not shown in the figure), at a value $\left\langle a^{2}\right\rangle^{1 / 2}=10^{-3}$. However, the behavior of the three simulations is again very similar, the slight discrepancies visible at the right end of the plot being due to a transient statistical fluctuation of the less populated simulation.

A deeper insight into the evolution of the velocity distribution can be obtained by constructing statistics concerning the relative velocity of particles as a function of their mutual distance $r$ at a given time: $\left\langle\Delta V_{\text {rel }}(r)\right\rangle$. The average is performed over all couples of particles inside the cluster. Calling $r_{\mathrm{ij}}$ the distance between particles $i$ and $j$, we can write:

$$
\left\langle\Delta V_{\mathrm{rel}}(r)\right\rangle=\frac{\sum_{r_{i j} \in(r, r+\Delta r)}^{N(r, \Delta r)}\left|\boldsymbol{v}_{i}-\boldsymbol{v}_{j}\right|}{N(r, \Delta r)},
$$

where $\Delta r$ represents an appropriate binning over distances, and $N(r, \Delta r)$ is the number of particles contained in the bin at distance $r$. As can be seen in Fig. 4 (upper panel), initial conditions impose the linearity of $\left\langle\Delta V_{\text {rel }}(r)\right\rangle$. The gravitational scattering of particles gradually changes the slope, increasing velocity dispersion and gradually diminishing the dependence of $\left\langle\Delta V_{\text {rel }}(r)\right\rangle$ on $r$. Short times (corresponding to lower curves in the top panel) clearly show that velocity dispersions have the tendency to increase faster at small values of $r$, due to shortrange mutual perturbations. Also shown in Fig. 4 (lower panel) is the velocity correlation, at a given time, as a function of $r$, defined as:

$$
C(r)=\frac{\sum_{r_{i j} \in(r, r+\Delta r)}^{N(r, \Delta r)} \frac{2\left(\boldsymbol{v}_{i} \cdot \boldsymbol{v}_{j}\right)}{\boldsymbol{v}_{i}^{2}+v_{j}^{2}}}{N(r, \Delta r)},
$$

using the same notation as in Eq. (9). The correlation in initial conditions tends obviously to unity for vanishing distances. During the evolution, its value decreases at a rate that, again, is not dependent on the resolution.

To make a tentative interpretation of this result we must estimate the cumulative effect of all the encounters as a function of the model parameters.

The number of encounters with impact parameter between $b$ and $b+\mathrm{d} b$ during a time interval $\tau$ can be estimated by:

$\mathrm{d} N=\frac{\pi v_{\mathrm{rel}} \Sigma_{\mathrm{c}} \tau}{\sigma_{\mathrm{z}} m_{\mathrm{p}}} b \mathrm{~d} b$,

where $m_{\mathrm{p}}$ is the mass of a planetesimal and for simplicity $\sigma_{\mathrm{z}}=\left\langle z^{2}\right\rangle^{1 / 2}$ (we assume here $G=1$ in all computations). The change of velocity normal to the trajectory for each encounter is then written as:

$\left|\delta v_{\perp}\right| \simeq \frac{2 m_{\mathrm{p}}}{b v_{\text {rel }}}$

Considering that:

$m_{\mathrm{p}}=\frac{\pi \Sigma_{\mathrm{c}} R_{\mathrm{c}}^{2}}{N_{\mathrm{cl}}}$, 

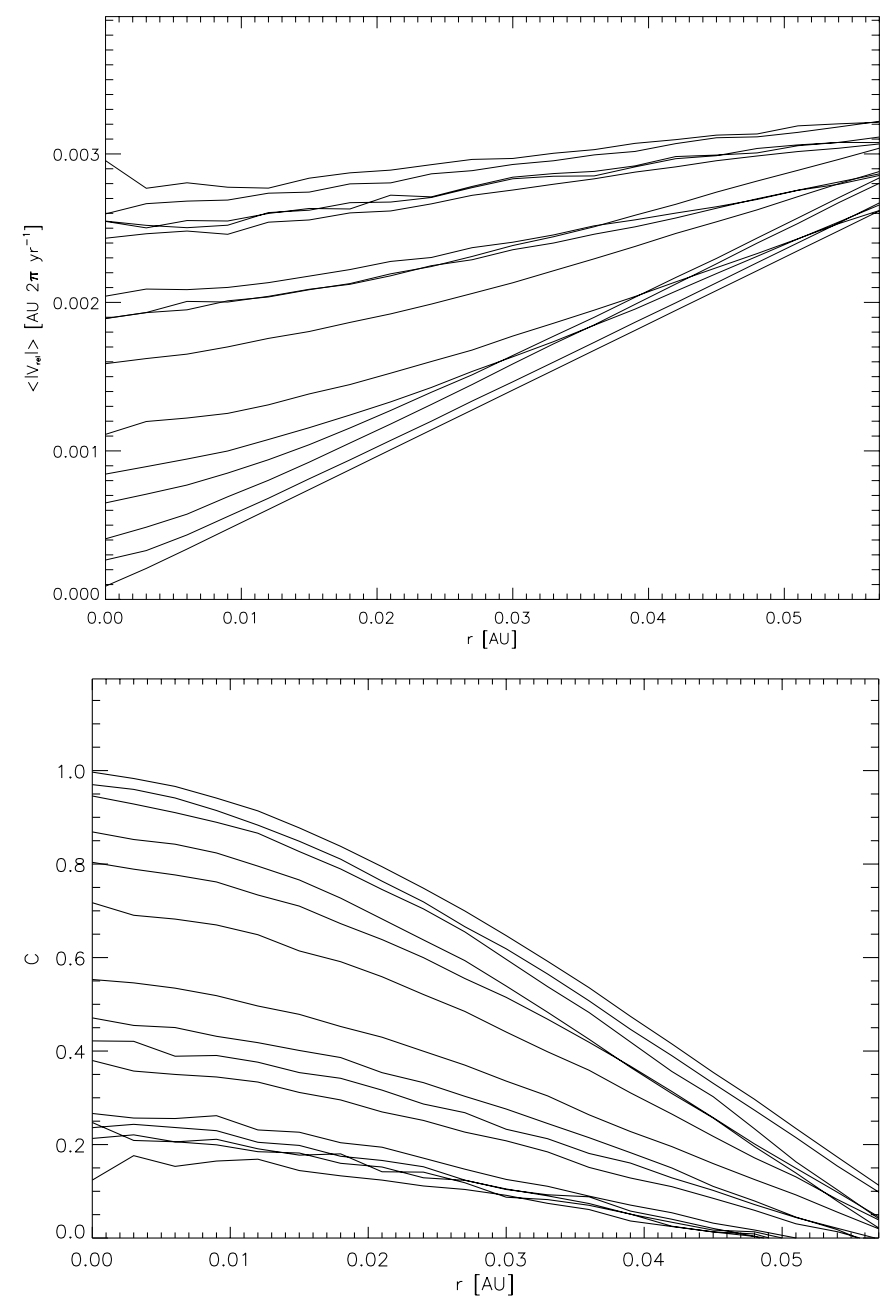

Fig. 4. Velocity difference (upper panel) and correlation (lower panel) between two particles as a function of their distance, for simulation 6 . Each quantity is averaged over all couples inside the cluster. Different curves correspond to different times. For the velocity difference, initial conditions are represented by the lower curve, showing a linear relation. Further evolution increases values and corresponds to curves having progressively shallower slopes. For the correlation, initial conditions correspond to higher values, reaching unity in the limit $r \rightarrow 0$. The time span represented here corresponds to the first four orbits.

and using again the same approximation as in (7) for $v_{\text {rel }}$ we obtain:

$\left|\delta v_{\perp}\right| \simeq \frac{2 \pi \Sigma_{\mathrm{c}} R_{\mathrm{c}}^{2}}{\Omega b^{2} N_{\mathrm{cl}}}$

The total velocity change in a time interval $\tau$ will then be given by:

$$
\begin{aligned}
\Delta v_{\perp}^{2} & =\int_{b_{\min }}^{b_{\max }}\left|\delta v_{\perp}\right|^{2} \mathrm{~d} N=\int_{b_{\min }}^{b_{\max }} \frac{4 \pi^{2} \Sigma_{\mathrm{c}}^{2} \tau}{\sigma_{\mathrm{z}} N_{\mathrm{cl}} \Omega b^{2}} \mathrm{~d} b \\
& =\frac{4 \pi^{2} \Sigma_{\mathrm{c}}^{2} \tau}{\sigma_{\mathrm{z}} N_{\mathrm{cl}} \Omega}\left(\frac{1}{b_{\min }}-\frac{1}{b_{\max }}\right) .
\end{aligned}
$$

Since $b_{\max }$ can be taken to be of the order of the largest scales of the system (for example $b_{\text {max }}=R_{\mathrm{cl}}$ ), the global behavior of the solution is in fact controlled by the choice of $b_{\min }$. Following

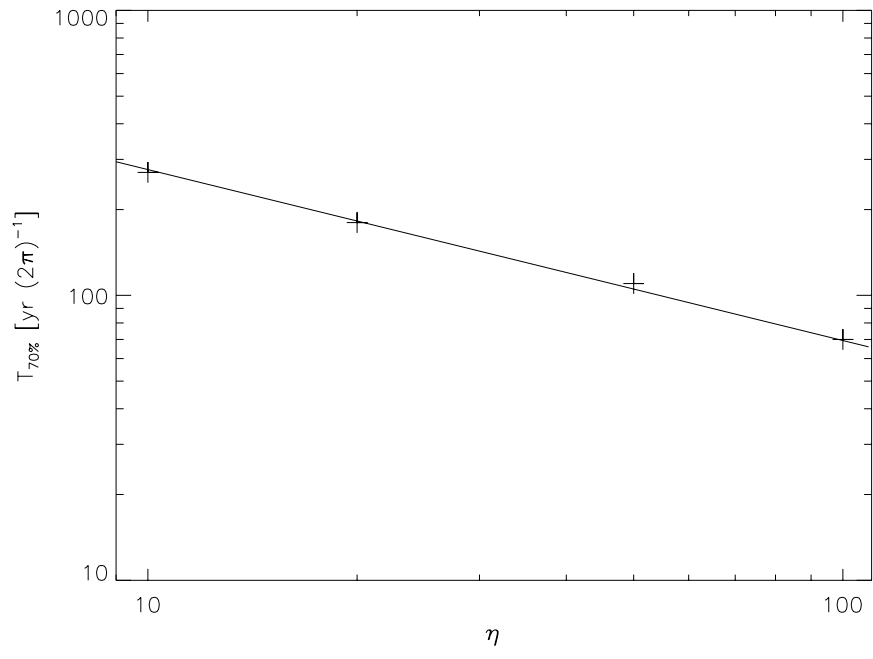

Fig. 5. The characteristic time $T_{70 \%}$ is plotted vs. $\eta$. The four points represent the values measured for simulations having identical number of particles (Nos. 3, 7, 8, 14). The result of the fit with the power law $T_{70 \%} \sim \eta^{-3 / 5}$ is shown by the straight line.

the choice by Athanassoula et al. (2001), we can take $b_{\min }$ to be the value of the impact parameter which would cause, on average, an angular deflection equal to $\pi / 2: b_{\min }=m_{\mathrm{p}} /\langle v\rangle^{2}$, where $\langle v\rangle$ represents an average value of the relative velocity. Expressing $m_{\mathrm{p}}$ as a function of $N_{\mathrm{cl}}$ as above, we observe that $b_{\text {min }} \sim 1 / N_{\mathrm{cl}}$. As a consequence, $\Delta v_{\perp}^{2}$ is not dependent on the resolution $N_{\mathrm{cl}}$. In fact, the enhanced encounter frequency resulting from an increase of $N_{\mathrm{cl}}$ is exactly compensated by the decrease in particle mass.

Nevertheless, the above analytical estimate is too simplicistic to capture the whole dynamics of the cluster.

A more careful - but complex - analysis, taking into account the evolution of the three-dimensional velocity dispersion and the gradual particle loss would better represent the dynamics of the clusters. In any case, we think that the result (16) offers a possible justification of the observation that, given the peculiar geometry and velocity profile, the dynamics is not closely related to the resolution.

Concerning the role of the density amplification, simulations Nos. 3, 7, 8, 14 suggest that a simple law relates $T_{70 \%}$ and $\eta$ (Fig. 5), of the kind:

$T_{70 \%} \sim \eta^{-3 / 5}$.

A dependence of $T_{70 \%}$ upon the initial vertical dispersion can be derived from simulations Nos. 3, 16, 17, 18. The decrease in mutual particle scattering with vertical dispersion is obvious, due to the reduction in collision frequency. At high dispersions a "saturation" of $T_{70 \%}$ is observed for $\left\langle z_{\mathrm{H}}^{2}\right\rangle^{1 / 2} \geq 30$. This is probably due to the onset of another regime, in which vertical velocities dominate and close encounters only happen in a restricted fraction of the orbit, close to the nodal intersections.

Concerning the role cluster size, it must be noted that a reduction in $R_{\mathrm{c}}$ has the effect of decreasing the role of the Keplerian shear, thus approaching the cluster to the limit of the gravitational instability. Since this regime determines a completely different behavior of the density fluctuations, its 
numerical study will be treated in detail in a separate paper. Here we just list in Table 1 some additional simulations (Nos. 20-23) giving the cluster dispersal time in a limited range of sizes. It can thus be seen that $T_{70 \%} \sim R_{\mathrm{c}}$. This can be explained as a geometric effect. In fact, as we verified in our results, the dispersion of the semi-major axis remains approximately the same. In this conditions, being the Keplerian shear the main dispersion mechanism, the dispersal time depends upon the distance that a particle has to travel in order to cross the cluster boundary. This distance is proportional to the cluster size, $R_{\mathrm{cl}}$, thus explaining the linear relationship above.

Finally, we have checked the dynamics of particles in some restricted domains inside the cluster. In particular we computed all the previous statistics in a more restricted elliptical area, having semi-axes of sizes $\alpha / 2$ and $\beta / 2$, i.e. in the central core of the over-dense region ( $25 \%$ of the original cluster area). All statistics concerning velocity dispersions and cluster dispersion remain essentially unchanged, thus hinting at a general homogeneity of the dynamics inside the cluster. Nevertheless, an initial transient increase in cluster concentration is detectable and especially prominent in the most massive ones. In fact, as can be observed in Fig. 2, a cluster can show the tendency to decrease its size during the first orbit. This transient phase suggests that the cluster is not at equilibrium and that self-gravity prevails for a short interval of time, during which the central region identified above reaches surface densities that are $\sim 4$ times larger than the original value of $\Sigma_{\mathrm{c}}$. This phase, anyway, does not last longer than a fraction of the first orbit, during which the transfer of gravitational potential into kinetic energy prevents any further collapse and induces the subsequent dispersion of particles.

\section{Complex initial velocity distributions}

The results presented above have been obtained by considering a particular velocity profile, defined in order to eliminate the initial contribution of the velocities of particles on the dispersion of the cluster. In fact, as already noted, in the absence of interparticle gravity the "ideal" velocity profile would provide elliptical patches of particles that never disperse. In this sense, we can say that cluster dispersion is the result of the action of gravity over an initially stable concentration of particles.

On the other hand, if the initial conditions corresponded to a velocity distribution deviating from the "ideal" profile, an additional source of cluster dispersion, independent from mutual gravitational interaction of particles, would be present. Of course, this is the situation that can be expected in general, since the velocity distribution of particles captured in a vortex will more or less deviate from the "ideal" one. As a consequence, in this case a purely gravitational evolution will induce cluster dispersion even if the role of scattering was negligible. In order to investigate the interplay of the two components (gravity plus a "non-ideal" initial velocity distribution), a more complex scenario, derived from the hydrodynamical simulation of an entire disk, was employed.

In fact, the mechanism of trapping in vortices has been verified in several studies by different approaches. Bracco et al. (1999) showed that anti-cyclonic vortices are the natural outcome of the evolution of random vorticity perturbations of a Keplerian velocity profile. Vortices thus created evolve, interact and dissipate with the complex dynamics typical of bidimensional turbulence. Bracco et al. (1999) also integrated the trajectories of dust particles, showing that even in this chaotically evolving system, the particles are trapped on timescales of the order of one revolution period.

Under the hypothesis that planetesimals collided in high density regions, grew to larger sizes, and that the nebula dissipated, we investigated the purely gravitational evolution of planetesimals in the model of Bracco et al. (1999). In order to do that, the positions and velocities of particles after a given time (20 orbital periods at distance $r=5.5 \mathrm{AU}$ from the Sun) were extracted from their simulations and used as initial conditions for the $N$-body code. Particles outside the interval $4<r<6$ AU were rejected (Fig. 6).

We then performed two kinds of simulations in which particle velocities are assigned following two different criteria:

1. " $B$ " simulations: velocities taken from the simulations of Bracco et al. (1999), i.e. with the memory of the effect of the past presence of the fluid flow;

2. " $K$ " simulations: pure Keplerian velocities, corresponding to circular orbits at the distance of each particle.

The second option offers a possibility of comparison with a more "ordered" situation than the complex dynamics obtained in the $B$ simulations. Vertical dispersions were imposed to be, in both cases, equal to $\left\langle z_{\mathrm{H}}^{2}\right\rangle^{1 / 2}=3$ in order to compare results with other simulations presented in Sect. 3 .

In order to build a quantitative estimate of the dispersion times, we isolated some clusters to be studied in detail. It must be noted that clusters in simulations $B$ and $K$ differ by their values of $R_{\mathrm{c}}, a_{\mathrm{c}}$ and $\eta$ from those studied in previous sections. We thus ran both the evolution of the cluster starting with the $K$ or the $B$ profile, and a small set of simulations of idealized clusters, having the corresponding characteristic parameters.

Our simulations clearly show that, in all cases, the concentrations of planetesimals dissipate faster than the previous "idealized" clusters. This result could be expected since the velocity profile of each cluster does not correspond to the profile of the idealized clusters.

We show here the results concerning one of these concentrations; they are representative of the behavior observed for all other selected clusters. The cluster studied here is visible in Fig. 6, (left panel) around coordinates $x=1.8, y=-5.3$. In order to improve statistics, the two-dimensional density field of the particle distribution was first reconstructed on a square grid, and then re-sampled using the "Triangular Shaped Cloud" (TSC) method, that ensures continuity of values and first derivatives of the field (Hockney et al. 1988). By a resampling of the density field, the total number of particles was thus increased to $6 \times 10^{4}$. Since we are not interested here in the evolution of small filaments of particles, the smoothing induced by the field reconstruction method is not relevant.

Figure 6 (right panel) shows the resulting particle distribution in the region of the cluster, in a local coordinate frame equivalent to that of Fig. 1 . The cluster has size $R_{\mathrm{cl}} \sim 0.4 \mathrm{AU}$, 

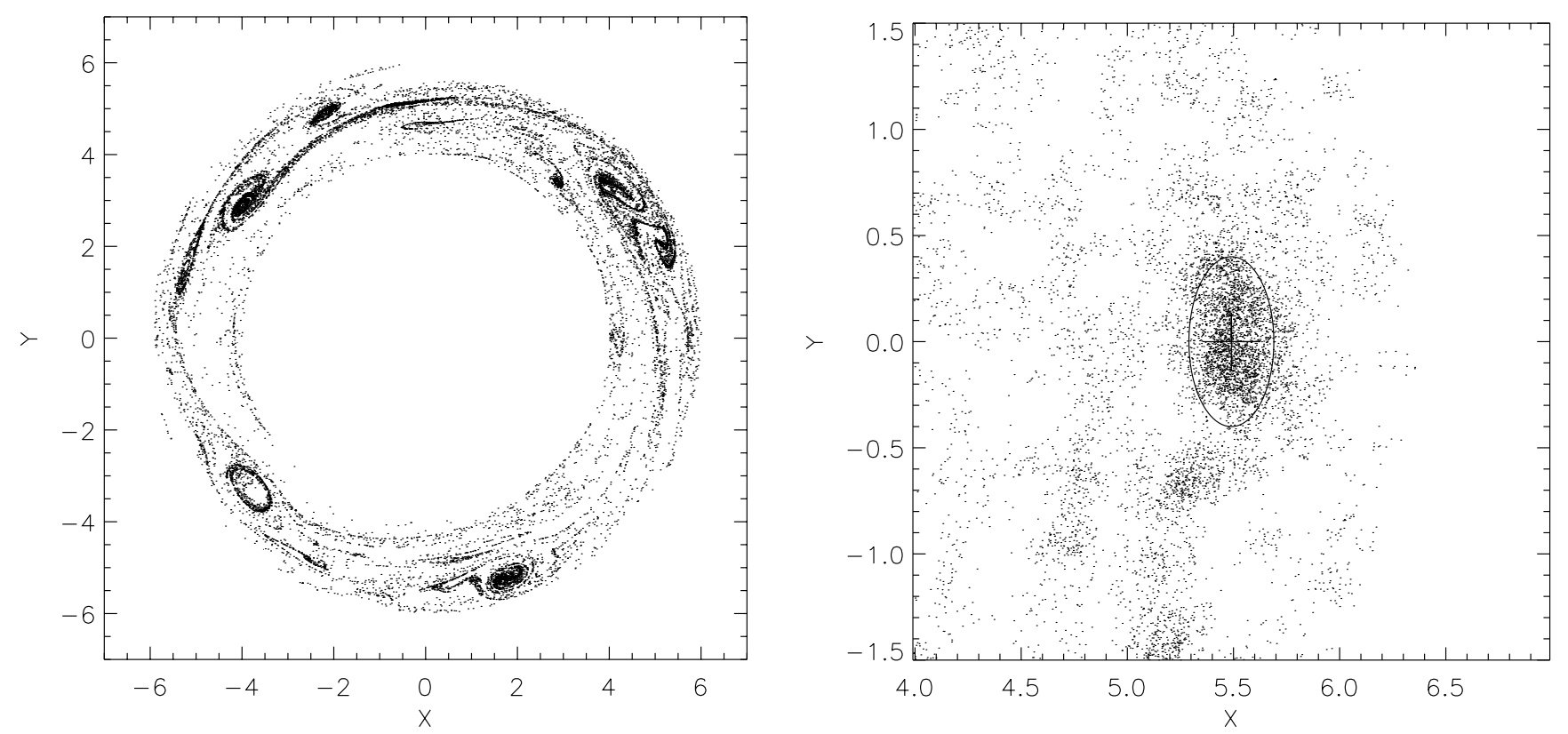

Fig. 6. Final state of the simulation of about 17000 particles in the presence of vortices after 20 orbits of the disk (at a distance of 5.5 AU from the Sun), obtained by Bracco et al. (1998). The plotted positions (left panel) were used as initial conditions for the gravitational evolution in the absence of gas drag. The right panel shows the region of the selected cluster after resampling.

its average distance from the Sun is $a_{\mathrm{c}}=5.49 \mathrm{AU}$, and its surface density corresponds to $\eta=15$ assuming $\Sigma_{\mathrm{d}}=1.2 \mathrm{~g} \mathrm{~cm}^{-2}$. As in Sect. 3, we studied the gravitational evolution of the cluster. For comparison, we ran a similar simulation using an idealized cluster having exactly the same $\eta, a$ and $R_{\mathrm{cl}}$, whose elliptical contour is represented in Fig. 6 (right panel).

The two cases were repeated both with and without the background disk, represented by particles whose initial positions, projected on the $(x, y)$ plane, fall outside the ellipse of Fig. 6 (right panel). A qualitative inspection of the cluster evolution reveals no apparent relevance of the surrounding disk, since interactions with other large density fluctuations happen well after the dispersal over a large area of the original condensation. The advantage of the isolated cluster is to allow an easier quantitative analysis of the dispersion rate (Fig. 7).

A comparison between $B, K$ cases and the "ideal" simulation clearly shows that gravity is not the only cause of dispersion of the cluster of planetesimals shown in Fig. 6 (right panel). The concentration decay of $B$ is characterized by a rapid fall (faster than that of the "ideal" case) at the beginning of the evolution, thus pointing out the role of an initial velocity distribution far from that of the "idealized" cluster. Nevertheless, initial velocities impose the signature of the anti-cyclonic vortex that trapped particles in this region. The result is a dispersion still slower than in the case of the purely Keplerian profile used in $K$.

\section{Conclusions}

We studied the dynamical evolution of an idealized, selfgravitating cluster, composed of equal-sized particles, whose initial velocity profile was chosen to allow a direct examination of the long-term stability of the structure. Its density amplification in comparison to the background density (which is

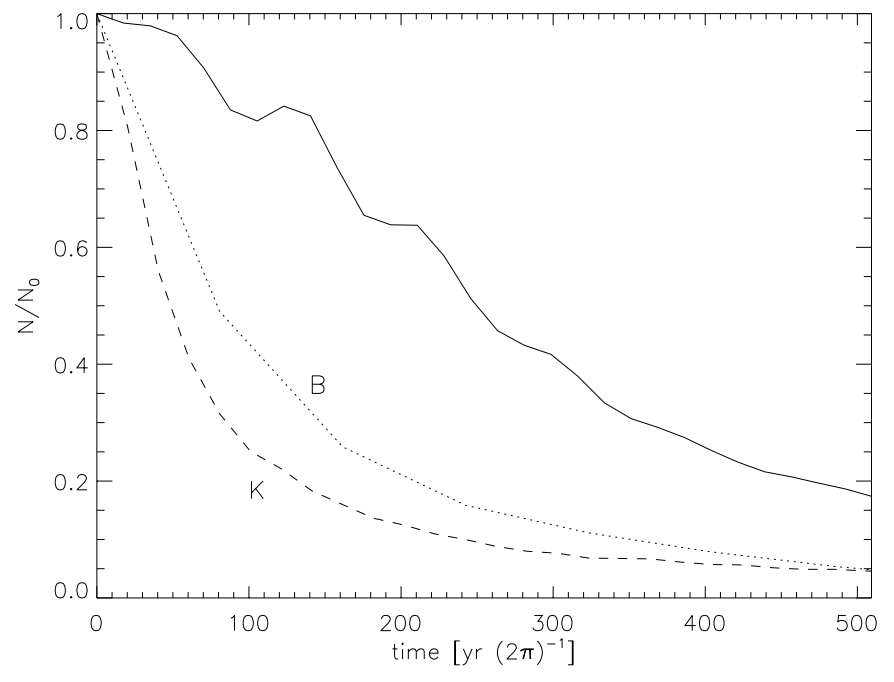

Fig. 7. A comparison between the dispersion rate of the cluster in Fig. 6 and an ideal cluster similar in size, in its distance from the Sun and in density (solid line). The two lines correspond each to the different velocity assignments of cases $K$ and $B$ (see text). Even if the "ideal" case is the most long-lived, the $B$ case preserves the cluster longer than in the $K$ simulation, thanks to the velocity pattern imposed by the vortex.

considered to be uniform) was high $(\eta>10)$, and its vertical dispersion was of the order of the Hill radius of the particles.

Our results indicate that:

1. Mutual perturbations induce a dispersion in semi-major axis values, that in turn allows Keplerian shear to gradually extract the particles from the region of the original cluster.

2. The cluster dispersion is not dependent on the size of the planetesimals, over a large range of masses (at least 
4 orders of magnitude), provided that the density amplification $\eta$ is constant.

3. A simple relation holds between the local density amplification and the dispersion rate of the cluster.

4. Denser clusters dissipate faster. In general, the dispersion of cluster components outside the original domain requires a few revolutions only.

5. The initial velocity of cluster members is critical in determining the evolution. The "ideal" clusters have allowed us to study the cluster dispersion with the effect of initial velocities minimized, i.e. under the effect of pure mutual gravity.

Due to the independence of these results from the resolution of the $N$-body simulations, it seems natural to extrapolate the conclusions to much smaller particles than those employed here, down to the size range in which vortex trapping is efficiently concentrating particles. Given a rigid profile such as that used as initial particle velocities, we can conclude here that, in the absence of gas, a local high concentration is rapidly dissipated. On the other hand, we want to underline that this does not exclude the possible existence of other velocity profiles capable of preserving the local concentration of at least some parts of the original cluster for a longer time. Nevertheless, even in the case of a cluster collapse under gravitational instability, we can state that a stable cluster configuration has not been reached. In general, velocity randomization has the tendency to disperse the cluster by de-correlating orbital parameters: a collapsing cluster could thus be gravitationally unstable on short timescales, such that it merges into a massive compact core before the velocity dispersion could destroy the initial concentration. Such an efficient collapse has not been observed in the range of parameters explored here.

Another mechanism capable of maintaining a compact shape of the cluster could be provided by the presence of gas. Gas drag, in fact, translates into the dissipation of the cluster kinetic energy, preventing an increase in velocity dispersions. This is particularly true inside a vortex, but it is strictly valid for a system of identical particles only, such as that studied here. In order for this process to be efficient, the capture time of the particle must be smaller that the typical timescales of the gravitational scattering. As shown by Chavanis (2000) the greatest capture efficiency is attained by particles strongly coupled to the gas, i.e. in the $1-100 \mathrm{~cm}$ diameter range. We can thus formulate a tentative scenario in which, at first, small particles are gradually concentrated inside vortices, where they begin to grow by mutual collisions. Later, they become large enough to decouple from gas, and they leave the vortex due to mutual perturbations. A detailed verification of this conclusion requires further simulations including the effects of both gravity and fluid drag, in the presence of coherent structures. This scenario will be the subject of a forthcoming paper.

It must also be noted that self-gravitating clusters of planetesimals, in presence of gravitationally unstable scale lengths, could have played a role in comets formation (Weidenschilling 1997). However, the cluster evolution in this dynamical regime is expected to be different from that dominated by the Keplerian shear, that we explored in this work. For this reason, we will devote a specific study to the numerical exploration of gravitationally instable disks of planetesimals in the comet formation regions.

Acknowledgements. Part of this work was supported by the Programme Nationale de Planétologie. We kindly acknowledge A. Bracco and collaborators for providing the outcome of their simulations, and G. Murante for his resampling code, Ch. Froeschlé for several useful discussions and the referee, S.J. Weindenschilling, for his helpful comments. We also wish to thank the IDRIS institute for the utilization of the parallel computers, T. Quinn for his help with the code pkdgrav, and the Observatoire de la Côte d'Azur (OCA) for the use of the SIVAM computer. One of the authors (P.T.) acknowledges the support of the H. Poincaré fellowship of the OCA.

\section{References}

Athanassoula, E., Vozikis, Ch. L., \& Lambert, J. C. 2001, A\&A, 376, 1135

Babiano, A., Boffetta, G., Provenzale, A., \& Vulpiani, A. 1994, Phys. Fluids, 6, 2465

Barge, P., \& Sommeria, J. 1995, A\&A, 295(1), L1

Bracco, A., Chavanis, P. H., Provenzale, A., \& Spiegel, E. A. 1999, Phys. Fluids, 11, 2280

Bracco, A., Provenzale, A., Spiegel, E. A., \& Yecko, P. 1998, in Proceedings of the Conference on Quasars and Accretion Disks, ed. M. A. Abramowicz (Cambridge Univ. Press, Cambridge), 254

Blum, J., \& Wurm, G. 2000, Icarus, 143, 138

Chavanis, P. H. 2000, A\&A, 356, 1089

Crisanti, A., Falcioni, M., Provenzale, A., Tanga, P., \& Vulpiani, A. 1992, Phys. Fluids A, 4, 1805

Cuzzi, J. N., Dobrovolskis, A. R., \& Champney, J. M. 1993, Icarus, 106,102

Cuzzi, J. N., Dobrovolskis, A. R., \& Hogan, R. C. 1993, in Chondrules and the Protoplanetary Disk, ed. R. Hewins, R. Jones, \& E. R. D. Scott (Cambridge Univ. Press, Cambridge)

Cuzzi, J. N., Hogan, R. C., Paque, J. M., \& Dobrovolskis, A. R. 2001, ApJ, 546(1), 496

Dobrovolskis, A. R., Cuzzi, J. N., \& Hogan, R. C. 1993, Bull. Am. Astron. Soc., 25, 1122

Hockney, R. W., \& Eastwood, J. W. 1988, Computer simulations using particles (Adam Hilger, Bristol)

Godon, P., \& Livio, M. 2000, ApJ, 537(1), 396

Goldreich, P., \& Ward, W. R. 1973, ApJ, 183, 1051

Klahr, H., \& Henning, T. 1997, Icarus, 128, 213

Murray, C. D., \& Dermott, S. F. 1999, Solar System Dynamics (Cambridge Univ. Press, Cambridge)

Richardson, D. C., Quinn, T., Stadel, J., \& Lake, G. 2000, Icarus, 143, 45

Safronov, V. S. 1969, Evolution of the protoplanetary cloud and formation of the Earth and the planets (Nauka, Moskow)

Squires, K. D., \& Eaton, J. K. 1991, Phys. Fluids A, 3, 1169

Tanga, P., Babiano, A., Dubrulle, B., \& Provenzale, A. 1996, Icarus, 121,158

Ward, W. R. 1976, in Frontiers of Astrophysics, ed. E. H. Avrett (Harvard Univ. Press, Cambridge)

Weidenschilling, S. J. 1995, Icarus, 116, 433

Weidenschilling, S. J. 1997, Icarus, 127, 290

Wisdom, J., \& Tremain, S. 1988, Astr. J., 95, 925

McWilliams, J. C. 1990, J. Fluid Mech., 219, 361 